\title{
Response in Target Lesion
}

National Cancer Institute

\section{Source}

National Cancer Institute. Response in Target Lesion. NCI Thesaurus. Code C94534.

A qualitative or quantitative measurement of the response of a target tumor(s) to the therapy. 